\title{
Editorial
}

\section{Nontraditional Therapy of Diabetes and Its Complications}

\author{
Ruozhi Zhao $\mathbb{D}^{1},{ }^{1}$ Amy Leung Hui $\mathbb{D}^{1},{ }^{1}$ Yong Xu $\mathbb{D}^{2},{ }^{2}$ and Michał Rabijewski $\mathbb{D}^{3}$ \\ ${ }^{1}$ Department of Internal Medicine, University of Manitoba, Winnipeg, Manitoba, Canada R3E 3P4 \\ ${ }^{2}$ Southwest Medical College, Luzhou 646000, China \\ ${ }^{3}$ St. Sophia Hospital, Warsaw 01-004, Poland \\ Correspondence should be addressed to Ruozhi Zhao; ruozhi.zhao@umanitoba.ca
}

Received 9 October 2020; Accepted 9 October 2020; Published 10 February 2021

Copyright (C) 2021 Ruozhi Zhao et al. This is an open access article distributed under the Creative Commons Attribution License, which permits unrestricted use, distribution, and reproduction in any medium, provided the original work is properly cited.

At present, the most traditional treatment for diabetes is still oral hypoglycaemic drugs and insulin therapy. Nontraditional treatment methods include nondrug therapy, unique treatments, emerging treatments, and application of natural hypoglycaemic drugs and ingredients. Nondrug therapy, such as exercise, nutrition, diet control, and psychotherapy, has achieved certain effects in some patients but has not attracted enough attention. In some countries, such as China and India, there are some effective and unique treatment methods in use, but these are not widely recognized and promoted. These emerging treatment methods include gene therapy, surgical treatment, and gut microbiota regulation therapy.

Traditional treatment methods have played an essential role in the treatment of diabetes but, at the same time, have various defects. Alternative and more effective treatments need to be searched, explored, and studied, particularly in nature, where there are many substances that are conducive to the prevention and treatment of diabetes. For example, in recent years, Amelanchier alnifolia, Dillenia indica, and Chrysophyllum albidum have been found to have significant antidiabetic effects, with further research pending.

Our special issue focuses on nontraditional treatment and application of diabetes patients and their complications, to improve efficacy, reduce side effects, improve patients' quality of life, and reduce the cost of medical care. In this special issue, we selected multiple original articles and eight reviews which are aimed at exploring the nontraditional therapy of diabetes and its complications from clinical and basic research aspects.
Painful diabetic peripheral neuropathy $(\mathrm{pDPN})$ is a debilitating complication of diabetes. Y. Zheng et al. searched through the medical records of all diabetic patients from January 2006 to January 2012; they identified and enrolled 30 moderate and severe $\mathrm{pDPN}$ patients in the study, for whom the treatment of neuropathic pain by regular pharmacotherapy had failed. The modified Huangqi Guizhi Wuwu Decoction (HGWD) treatment was administered orally twice a day for 6 months. The numerical rating scale (NRS) level at month 6 was $2.57 \pm 2.30$, significantly improved compared with the baseline level of $6.03 \pm 1.83(p<0.05)$. The amelioration of 3 common symptoms, namely, limb pain, limb numbness, and insomnia, was evident, and the major response of common symptoms at month 6 including limb pain, insomnia, and limb coldness was significantly increased compared with the results at month $3(p<0.05)$. Moreover, 2-hour postprandial blood glucose (2hPG) level decreased from $10.77 \pm 1.29 \mathrm{mmol} / \mathrm{L}$ at baseline to $9.66 \pm 0.60 \mathrm{mmol} /$ $\mathrm{L}$ at month $6(p<0.05)$. No serious adverse events occurred throughout the treatment period. Ginseng roots have been widely used as an herbal treatment in East Asia for more than 2000 years. Korean Red Ginseng (KRG) is a popular traditional herb that is known to have beneficial effects in obesity, postmenopausal symptoms, cholesterol levels, and cardiovascular diseases. Many type 2 diabetes mellitus (T2DM) patients in Korea take KRG for various reasons. K. Park et al. investigated the effects of KRG administration on diabetic peripheral neuropathy in T2DM patients. Sixty-one patients completed the study. The current perception threshold (CPT) of the lower extremities at various frequencies 
exhibited significant improvements at week 24 in the KRG group. Fasting plasma glucose, $\mathrm{HbAlc}$, and various metabolic and inflammatory markers were not altered after 24 weeks in both groups. In the subgroup analysis, CPT levels were improved in those with a longer diabetes duration or who already had neuropathy at the beginning of the study, and insulin resistance was improved in patients with a shorter diabetes duration.

Because low-carbohydrate diets are effective strategies to improve insulin resistance, the hallmark of type T2DM, C. $\mathrm{M}$. Walton et al. revealed the meaningful changes observed in 90 days of low-carbohydrate (LC) ketogenic dietary intervention in female type T2DM aged 18-45. Eleven women who were recently diagnosed with T2DM volunteered to participate in an intensive dietary intervention to limit dietary carbohydrates to under 30 grams daily for 90 days. The volunteers lost significant weight $(85.7 \pm 3.2 \mathrm{~kg}$ to $76.7 \pm 2.8 \mathrm{~kg}$ ) and lowered systolic ( $134.0 \pm 1.6$ to $123.3 \pm 1.1 \mathrm{mmHg}$ ) and diastolic $(89.9 \pm 1.3$ to $82.6 \pm 1.0 \mathrm{mmHg})$ blood pressure. HbA1c dropped to 5.6\%. Most blood lipids were significantly altered, including HDL cholesterol $(43.1 \pm 4.4$ to $52.3 \pm 3.3 \mathrm{mg} / \mathrm{dL})$, triglycerides $(177.0 \pm 19.8$ to $92.1 \pm 8.7$ $\mathrm{mg} / \mathrm{dL})$, and the TG:HDL ratio $(4.7 \pm 0.8$ to $1.9 \pm 0.2)$. LDL cholesterol, AST, and ALT were not significantly different. The role of traditional Chinese medicine (TCM) in diabetes mellitus (DM) treatment has been recognized widely, while there are few long-term observation reports. X. Wei et al. introduced a case of DM treated by classical prescription alone for 10 years, which would provide the reference for clinical practice. A 64-year-old male was treated with 10 years of continuous classical prescription alone. After 3 months of TCM treatment, the patient's blood glucose level decreased significantly and blurred vision symptoms improved. With continued TCM treatment, his urinary albumin excretion rate (UAER) normalized. Subsequently, he continued the outpatient consultation, and his TCM prescription was adjusted according to clinical symptoms. After 10 years of continuous TCM treatment, his blood glucose remained stable, urinary microalbumin quantitation showed no abnormalities, and blurred vision disappeared. Diabetic nephropathy (DN) is the leading cause of end-stage renal disease (ESRD). The ROS-mediated PI3K/AKT pathway plays a key role in podocyte apoptosis and DN progression. Baoshenfang (BSF), as a common TCM compound, has been widely used in the treatment of DN in clinical practice. BSF consists of a group of herbal medicines including Astragalus membranaceus, Rehmannia, Salvia miltiorrhiza Bunge, Cuscuta chinensis, Herba Artemisiae Anomalae, Euonymus alatus, and Hirudo. F. Cui et al. reported that Baoshenfang (BSF) significantly decreased $24 \mathrm{~h}$ urinary protein, serum creatinine, and blood urea nitrogen levels in DN mice. Meanwhile, BSF significantly inhibited oxidative stress and podocyte apoptosis in their in vivo and in vitro studies. Moreover, BSF significantly decreased the inhibition of the PI3K/AKT pathway induced by high glucose (HG) in DN. More importantly, the effects of BSF on podocyte apoptosis were reversed by PI3K siRNA transfection.

Carrageenan intake in mice led to marked glucose intolerance and insulin resistance due to stimulation of systemic inflammation and inhibition of insulin signalling. L. Feferman et al. demonstrated that participants with prediabetes on no carrageenan had declines in HbAlc and HOMA-IR ( $p=0.006$ and $p=0.026$; paired $t$-test, two-tailed). They had increases in C-peptide $(p=0.029)$ and Matsuda index $(2.1 \pm 0.7$ to $4.8 \pm 2.3 ; p=0.052)$ and declines in serum IL8 , serum galectin-3, and neutrophil phosphor (Ser307/312)IRS1 $(p=0.049, p=0.003$, and $p=0.006$; paired $t$-tests, two-tailed). Subjects on the diet with carrageenan had no significant changes in these parameters. Significant differences between no-carrageenan and carrageenan-containing diet groups for changes from baseline to 12 weeks occurred in C-peptide, phospho-Ser-IRS1, phospho-AKT1, and mononuclear cell arylsulfatase B $(p=0.007, p=0.038, p=0.0012$, and $p=0.0008 ; 2 \times 2$ ANOVA). Significant correlations were evident between several of the variables. Ginsenoside is a major pharmacological active ingredient of ginseng. Ginsenoside $\mathrm{Rg} 3$ monomer is the monomer saponin extracted from red ginseng. There are two isomers, $20(\mathrm{R})$-ginsenoside Rg3 (20(R)-Rg3) and 20(S)-ginsenoside Rg3 (20(S)-Rg3). $20(\mathrm{~S})-\mathrm{Rg} 3$ has been reported to have anticancer and antidiabetic effects. T. Zhou et al. observed that the change in renal histology was improved and renal tubular epithelial cell apoptosis decreased significantly by treatment with 20 (S)-Rg3 in diabetic rats. Furthermore, the urine protein decreased in the rats with the 20(S)-Rg3 treatment. Fasting blood glucose, creatinine, total cholesterol, and triglyceride levels in the 20(S)$\mathrm{Rg} 3$ treatment group were all lower than those in the diabetic group. 20(S)-Rg3 dramatically downregulated the expression of TGF- $\beta 1$, NF- $\kappa$ B 65 , and TNF- $\alpha$ in the kidney. These resulted in a significant prevention of renal damage from inflammation. Patients with diabetes mellitus are at twice the risk of developing depression than the general population. The coexistence of diabetes and depression largely contributes to increased morbidity and mortality and results in high healthcare costs. B. B. Gebre et al. assessed the severity of depression and its determinants in diabetes outpatients at Hawassa University Comprehensive Specialized Referral Hospital, Southern Ethiopia. The results indicated that the magnitudes of depression were found to be $41.5 \%$. The potential predictors were adhering to alcohol intake (adjusted odds ratio, $\mathrm{AOR}=3.71,95 \% \mathrm{CI}: 1.52,9.06$ ), loss of someone very close or spouse (AOR $=6.83,95 \% \mathrm{CI} 3.07$, 15.19), having no social support ( $\mathrm{AOR}=3.68,95 \% \mathrm{CI}$ : 1.63 , 8.29), not adhering to the recommended dietary regimen $(\mathrm{AOR}=6.83,95 \% \mathrm{CI} 3.07,15.19)$, not adhering to physical activity ( $\mathrm{AOR}=4.1,95 \% \mathrm{CI}: 1.86,9.014$ ), not adhering to medication $(\mathrm{AOR}=4.2,95 \% \mathrm{CI}: 1.7,10.31)$, and not having raised blood pressure of $140 / 90 \mathrm{mmHg}$ and above $(\mathrm{AOR}=7.42,95 \% \mathrm{CI}: 3.40,16.17)$. Nonpharmaceutical therapies such as exercise training and diet intervention are widely used for the treatment of insulin resistance (IR). The skeletal muscle is the major peripheral tissue of glucose metabolism under insulin stimulation. Using a high-fat diet-induced IR mouse model, J. Qi et al. showed that NADPH oxidase 4 (Nox4) upregulation mediates the production of reactive oxygen species (ROS) that causes metabolic syndrome featuring IR. The Nox4 expression level was markedly elevated in IR mice, and Nox4 overexpression 
was sufficient to trigger IR. Conversely, downregulation of Nox4 expression through exercise training prevented dietinduced IR by reducing the production of ROS and enhancing the AKT signalling pathway. No study has investigated the role of induced membrane (IM) formation in treating diabetic foot ulcer (DFU). C. Liu et al. evaluated the potential role of a two-staged surgical approach, comprising polymethyl methacrylate (PMMA) implantation and IM formation, in the treatment of DFU, comparing the results of those with routine wound debridement in patients with DFUs and nonrevascularized peripheral arterial disease (PAD). They demonstrated that in the PMMA group, 16 patients $(57.1 \%)$ with intact IM formation achieved ulceration healing at $13.1 \pm 3.7$ weeks with a mean number of debridements of $1.3 \pm 0.4$, which were significantly different compared to those values in 5 patients of the conventional group $(22.7 \%, p=0.014$; healing duration: $26.4 \pm 7.8$ weeks, $p=0.016$; mean number of debridements: $3.6 \pm 0.5, p \leq$ 0.001 ). At a mean $16.8 \pm 4.3$-month follow-up, patient survival rates were $92.9 \%$ and $68.2 \%$ in the PMMA and conventional groups, respectively $(p=0.032)$. The major amputation rate and reulceration of DFUs were similar between the groups. X. Zhao et al. investigated the effect of a peer support education model on the levels of glucose and lipids in patients with T2DM in China. 120 T2DM patients were assigned to two groups randomly. Group 1 was trained on basic diabetic medical knowledge by a professional medical staff. After 8 weeks of studying, these specially trained patients then trained the patients of Group 2. It was found that after 3 months of intervention, the levels of fasting blood glucose, triglycerides, glycosylated haemoglobin, total cholesterol, and low-density lipoprotein cholesterol were significantly decreased in the two groups $(p<0.05)$. However, with the prolongation of time, there were no significant changes in the two groups in the above indices $(p>0.05)$ after 6 months, and the result was the same after stratifying for sex, age, and education level. Meanwhile, there was no significant difference in decreasing glucose and lipid levels between the two groups' participants $(p>0.05)$. Y. Luo et al. developed a decision support software for healthcare professionals in type 2 diabetes. It was an independent software on a tablet to record the data of patients and treatments given by their physicians. A major function of the software was to remind doctors when and how they should implement the standard care as recommended by the Chinese Diabetes Society guideline. Secondly, they compared the baseline data of standard care including statin and aspirin usage with data from a previous " $3 \mathrm{~B}$ study" to see whether there was an improvement of these standard cares. Finally, they further compared the data during four quarters of the whole year to evaluate whether there was a continuous improvement. During the first quarter, 27,291 cases and 27,352 cases were collected with complete information about statin and aspirin usage, respectively. The percentage of patients treated with statins and aspirin in their study was significantly higher than that reported in the $3 \mathrm{~B}$ study (59.6\% vs. $19.9 \%$ and $59.8 \%$ vs. $18.5 \%, p<0.001)$. There were no significant differences among the four quarters for the percentage of patients who were taking statins or aspirin $(p>0.05)$. Distal sensory- motor polyneuropathy is one of the most frequent diabetic complications. S. Asano et al. revisited the polyol pathway, investigating the curative effects of a novel strong aldose reductase inhibitor, ranirestat, in streptozotocin-induced diabetic rats with preexisting polyneuropathy. They demonstrated that sensory nerve conduction velocity increased in diabetic rats treated with ranirestat $(43.3 \pm 3.6 \mathrm{~m} / \mathrm{s})$ compared with rats treated with placebo $(39.8 \pm 2.3)$. Motor nerve conduction velocity also increased in the ranirestat group $(45.6 \pm 3.9)$ compared with the placebo group $(38.9 \pm 3.5)$. The foot withdrawal latency to noxious heating was improved in the ranirestat group $(17.7 \pm 0.6$ seconds) compared with the placebo group $(20.6 \pm 0.6)$. The decrease in the intraepidermal fibre density was significant in the diabetic placebo group $(21.6 \pm 1.7 / \mathrm{mm})$ but not significant in the diabetic ranirestat group $(26.2 \pm 1.2)$ compared with the nondiabetic placebo group $(30.3 \pm 1.5)$. Neurite outgrowth was promoted in neurons supplemented with ranirestat (control $1446 \pm 147 \mu \mathrm{m} /$ neuron, ranirestat $2175 \pm 149$ ). Post transplantation diabetes mellitus (PTDM) is a known complication of transplantation that affects the prognosis. Tacrolimus is a widely used immunosuppressant that has been reported to be a risk factor for PTDM and to further induce complications in heart and skeletal muscle. C. Zheng et al. found that the quadriceps in the tacrolimus-induced PTDM group were smaller than those in the control group. The study identified 275 differentially expressed genes (DEGs) that may be responsible for insulin resistance and the progression of PTDM, including 86 upregulated genes and 199 downregulated genes. GO and KEGG functional analyses of the DEGs showed a significant correlation between PTDM and muscle development. PPI network analysis screened eight hub genes and found that they were related to troponin and tropomyosin. Diabetic cardiomyopathy is a common cardiac condition in patients with diabetes mellitus, which results in cardiac hypertrophy and subsequent heart failure. Accumulated evidence implicates pyroptosis as a vital contributor to the hyperglycaemia-induced cardiac inflammatory response. Exendin-4, a GLP analogue, promotes the survival of cardiomyocytes in cardiovascular diseases, including diabetic cardiomyopathy. $\mathrm{H}$. Wei et al. revealed that Exendin-4 treatment protected against heart remoulding and dysfunction and attenuated cardiac inflammation in high-fat diet-fed rats. They identified Exendin-4 as a pyroptotic inhibitor protecting against hyperglycaemia-induced cardiomyocyte pyroptosis via the AMPK-TXNIP pathway. Y. Zhang et al. investigated the changes of renal structure and its function in normal glucose tolerance (NGT), impaired glucose tolerance (IGT), DM, and diabetic kidney disease (DKD) stages in OLETF rats and explored the role of the INS/IRS-1/PI3-K/Akt signalling pathway. In the IGT group, the index which reflects the function of renal tubulelike $\mathrm{N}$-acetyl- $\beta$-glucosaminidase, neutrophil gelatinaseassociated lipocalin, retinol-binding protein, and cystatin $\mathrm{C}$ was higher than those in the control group and the NGT group $(p<0.05)$. Significant renal structure damage, especially in renal tubules, was observed in the IGT group. In the presence of insulin at a high concentration, the IRS1/PI3-K/Akt signalling pathway in renal tubular epithelial 
cells was inhibited, and the expression of megalin and cubilin was significantly downregulated which was accompanied by a minimum uptake of albumin.

In a review article, in response to the recent rise in the numbers of diabetes patients, many treatments have been developed, but currently, oral antihyperglycaemic agents and insulin are still the main clinical treatments. Since current drugs have limitations and harmful side effects, research into alternative treatments has been sought. L. Zhao et al. reviewed recent research updates of Saskatoon berries (SB), covering its background information, its main active ingredients, its structure, and its function. Flavonoid compounds in Saskatoon berries, in particular flavanol, anthocyanin, and proanthocyanidin, possess anti-inflammatory, antitumor, and antidiabetic impacts. They synthesized the latest research on the health benefits of Saskatoon berry in a variety of domains. With further research, SB has the potential to help treat and prevent diabetes in the future. Gestational diabetes mellitus is the most common metabolic disorder during pregnancy with health consequences for both lives during and after pregnancy. With the growing popularity of traditional Chinese medicine, Y. X. Z. Xu et al. found three major herbal medicines/herbal products that were associated with glycaemic control in gestational diabetes, including Zuo Gui Wan, red raspberry leaves, and Orthosiphon stamineus. They further reviewed them and their relatives in relation to T2DM and found more evidence of metabolic benefits. None of the herbal medicines and products examined reported toxicity in the experimental models. Overall, treatments of gestational diabetes by western or alternative interventions are grossly understudied. It is critical to have a standardized protocol when evaluating efficacy of herbal medicines and produce quality results for women and their health care providers to make informed treatment decisions. Herbal plants are the richest source of drugs in India from prehistoric times. P. Kamboj et al. reviewed a less explored medicinal plant, Dillenia indica, which has promising effects in treating diabetes and other diabetic-associated complications. Despite its wide use in the northeast region of India as a traditional medicine, there is only one clinical study where the antidiabetic potential of the fruit powder has been shown. Further well-designed animal and human studies are needed to confirm the role of Dillenia indica in diabetes and its associated complications. Y. S. Huang et al. investigated the efficacy of a very low-energy diet (VLED) in overweight and obese individuals with T2DM. Results indicated that eighteen studies (11 randomized and 7 nonrandomized controlled trials) with 911 participants were included. The metaanalyses showed that compared with a low-energy diet (LED) and mild energy restriction (MER), VLED is superior in the reduction of body weight (mean difference $(\mathrm{MD}) \mathrm{MD}_{\mathrm{LED}}=$ $-2.77,95 \%$ confidence interval $(\mathrm{CI}) \mathrm{CI}_{\mathrm{LED}}=-4.81$ to -0.72 , $P_{\mathrm{LED}}=0.008 ; \quad \mathrm{MD}_{\mathrm{MER}}=-6.72, \quad 95 \% \quad \mathrm{CI}_{\mathrm{MER}}=-10.05$ to $\left.-3.39, P_{\mathrm{MER}}<0.0001\right)$, blood glucose $\left(\mathrm{MD}_{\mathrm{LED}}=-1.18,95 \%\right.$ $\mathrm{CI}_{\mathrm{LED}}=-2.05$ to $-0.30, P_{\mathrm{LED}}=0.008 ; \mathrm{MD}_{\mathrm{MER}}=-6.72,95 \%$ $\mathrm{CI}_{\mathrm{MER}}=-10.05$ to $\left.-3.39, P_{\mathrm{MER}}<0.0001\right)$, and triglyceride (TG) $\left(\mathrm{MD}_{\mathrm{LED}}=-0.35,95 \% \mathrm{CI}_{\mathrm{LED}}=-0.58\right.$ to $-0.12, P_{\mathrm{LED}}=$ $0.002 ; \mathrm{MD}_{\mathrm{MER}}=-0.55,95 \% \mathrm{CI}_{\mathrm{MER}}=-0.93$ to -0.17 , $\left.P_{\text {MER }}=0.005\right)$ levels at the end of the intervention. After the follow-up (1-5 years), no obvious difference in weight loss $\left(\mathrm{MD}=-0.84,95 \% \mathrm{CI}=-3.01\right.$ to $\left.1.32, p=0.45, I^{2}=0 \%\right)$ and TG level $(\mathrm{MD}=-0.25,95 \% \mathrm{CI}=-0.55$ to $0.06, p=0.12$, $\left.I^{2}=0 \%\right)$ between VLEDs and LEDs was evident, but VLED is more effective in glycaemic control $(\mathrm{MD}=-1.43,95 \%$ $\mathrm{CI}=-2.65$ to $-0.20, p=0.02$ ). Compared to bariatric surgery, VLEDs offered comparable effects on weight loss $(\mathrm{MD}=2.51,95 \% \mathrm{CI}=-9.52$ to $14.54, p=0.37)$, glycaemic control $(\mathrm{MD}=0.37,95 \% \mathrm{CI}=-0.22$ to $0.96, p=0.22)$, $\mathrm{TG}$ $(\mathrm{MD}=-0.3,95 \% \mathrm{CI}=-0.74$ to $0.17, p=0.7)$, and insulin resistance improvement $(\mathrm{MD}=-1,95 \% \mathrm{CI}=-2.7$ to 0.7 , $p=0.25$ ). The liver is regarded as an important link in the management and control of DM, including the alleviation of glucose metabolism and lipid metabolism and others via glucose storage and endogenous glucose generation from glycogen stored in the liver. Y. Zhao et al. reviewed the management of DM in patients with chronic liver disease. Liver cirrhosis is a widespread chronic disease, which often lowers the quality of life and decreases life expectancy. According to a growing body of research, diabetes shows a close correlation with hepatitis, liver cirrhosis, and liver cancer. Moreover, the coexistence of liver complications would accelerate the deterioration of patients with diabetes. Liver cirrhosis and diabetes influence each other. Thus, in addition to pharmacological treatments and lifestyle interventions, effective control of cirrhosis might assist in a better management of diabetes. In regard to different etiologies of liver cirrhosis, different therapeutic methods, such as antiviral treatment, may be more effective. Effective control of cirrhosis might be a strategy for better management of diabetes. Diabetes secondary to chronic pancreatitis (CP) or type $3 \mathrm{cDM}$ refers to a brittle form of diabetes and is often characterised by hypoglycaemic episodes, erratic glycaemic control, and impaired quality of life. It differs in other forms of diabetes and is typically characterised by concurrent pancreatic, endocrine, and exocrine insufficiency which can present as malabsorption and nutritional deficiencies. P. C. Johnston et al. discussed the pathogenesis, epidemiology, and practicalities of diagnosis, screening, and management of this condition. T2D is an independent risk factor of stroke and systemic embolism in patients with atrial fibrillation (AF), and T2D patients with AF-associated stroke seem to have a worse clinical outcome and a higher risk of unfavourable clinical course compared to individuals without this metabolic disorder. D. Prídavková et al. reviewed the current data regarding the use of direct oral anticoagulants (DOACs) in individuals with T2D and AF. Long-term anticoagulation is indicated in the majority of T2D patients with AF to prevent adverse AF-associated embolic events. DOACs, direct oral thrombin inhibitor dabigatran, and direct oral factor Xa inhibitors, rivaroxaban, apixaban, and edoxaban have emerged as a preferred choice for long-term prevention of stroke in AF patients offering potent and predictable anticoagulation and favourable pharmacology with low risk of interactions. S. Guo et al. undertook the systematic review and meta-analysis to summarize the effect of diacerein on T2DM. They found that five randomized controlled trials (RCTs) with 278 participants were included. Compared with controls, diacerein provided 
significant improvement on fasting blood glucose (FBG) (MD -0.52; 95\% CI (-0.89 -0.14); $p=0.007)$, but trial sequential analysis (TSA) showed that this positive effect required more support. Besides, diacerein also significantly improved HbA1c (MD -0.71; 95\% CI (-1.07 -0.36); $p<0.001$ ), BMI (MD -0.40; 95\% CI $(-0.49 \sim-0.31) ; p<0.001)$, and CRP level (MD -1.49; 95\% CI $(-2.78 \sim-0.19) ; p=0.02)$. No superiority was noted in favour of either treatment regarding lipid profiles or haematological indexes. Among all complications, diacerein caused significantly more gastrointestinal syndromes (RR 1.39; 95\% CI (1.08 1.77); $p=0.009)$.

The articles in this issue provide some new ideas for nontraditional therapy of diabetes and its complications. It is our hope that our readers will enjoy these articles and assist them in their future research.

\section{Conflicts of Interest}

The authors declare that they have no conflict of interest.

\section{Acknowledgments}

We are very grateful to all authors and reviewers. As guest editors, we are honoured to share this discussion with you.

Ruozhi Zhao

Amy Leung Hui

Yong Xu

Michat Rabijewski 